\title{
Wide Range High Precision CMOS Exponential Circuit Based on Linear Least Squares Approach
}

\author{
Ali NADERI SAATLO ${ }^{1}$, Serdar OZOGUZ ${ }^{2}$ \\ ${ }^{1}$ Dept. of Electrical-Electronics Engineering, Urmia Branch, Islamic Azad University, Urmia, Iran \\ ${ }^{2}$ Faculty of Electrical-Electronics Engineering, 34469, Maslak, Istanbul, Turkey \\ a.naderi@iaurmia.ac.ir,ozoguz@itu.edu.tr \\ Submitted March 12, 2018 / Accepted August 14, 2018
}

\begin{abstract}
A new strategy to implement exponential circuit in CMOS technology is presented in this paper. The proposed method is based on the new approximation function optimized by linear least squares approach to extend the output dynamic range. The current mode method is employed for realization of circuits, because of simple circuitry and intuitive topology. Unlike to the some reported circuits which were designed in the subthreshold region, the proposed design operates in the saturation region which provides acceptable bandwidth for the circuit. In order to validate the circuit performance, the post layout simulation results are presented using HSPICE and $\mathrm{Ca}$ dence with TSMC level 49 (BSIM3v3) parameters for $0.18 \mu \mathrm{m}$ CMOS technology. The results demonstrate $78 \mathrm{~dB}$ output dynamic range with the linearity error less than $\pm 0.5 \mathrm{~dB}$ which shows a remarkable improvement in comparison with previously reported works. A bandwidth of $67 \mathrm{MHz}$, maximum power consumption of $0.326 \mathrm{~mW}$ under supply voltage of $1.5 \mathrm{~V}$, and $0.77 \%$ error for temperature variations are further achievement of the design.
\end{abstract}

\section{Keywords}

Exponential function, new approximation, analog circuit, high precision

\section{Introduction}

Computational circuits are important building blocks which are utilized in various applications in the field of signal processing. Exponential, logarithm, multiplication, division, squaring, square rooting are main functions which realize a multitude of circuits with a lot of applications. Multiplier and divider circuits are very useful in telecommunication circuits, fuzzy controllers and neural networks. Exponential (EXP) circuits are employed in medical equipments [1], hearing devices [2] or disk drives [3]. Squaring circuits represent the basic cell for realizing any continuous function, using function synthesizer circuits [4], [5]. Square rooting circuits are used in RMS to DC converters, function synthesizer and fuzzy systems [6].

Among these computational circuits, Exponential
Function Generator (EXPFG) circuit is difficult to implement, because of its inherent nonlinear behavior. The importance of this function originates from the fact that it is utilized as a part of automatic gain controllers or variable gain amplifiers (VGA) [7], [8]. The most important aspects of EXPFG circuits include output dynamic range, linearity of the output in $\mathrm{dB}$, accuracy, power consumption, and area efficiency. In the following, the literature review verified that most of the above mentioned parameters have a trade off with each other, and then different design techniques have been presented [10-21] to satisfy the compromise between the characteristics.

Considering the types of transistors, two groups of implementation using BJT and MOS transistors exist in the literature: the first approach is based on using exponential characteristic of bipolar transistor to obtain exponential function. The main drawback of using bipolar transistor is the nonzero value of the base current, and also its dependency to the temperature which causes large errors in the resulted exponential function [9]. The second group is based on the MOS transistor which employs exponential current-voltage characteristic of the transistor in subthreshold region. However, the poor circuit frequency response and narrow input dynamic range are the disadvantages of this method, which originates from very small drain currents for charging and discharging of the transistor parasitic capacitances [10-13].

Considering the mathematical techniques, three methods have been presented. The first group emphasizes on the use of expansion of the EXP functions using Taylor series [14], [15]. The main weakness of this method is given by the relatively small output dynamic range, because of neglecting superior-order terms in Taylor series. In the case of using higher-order terms, accuracy will be increased as well as the output dynamic range; however complex circuits with more silicon area are required. Therefore a tradeoff between the overall circuit precision and the silicon area must be made.

The second group uses piecewise linear approximation method [16-18], which requires appropriate positioning of the breakpoints. The poor accuracy in the breakpoints as well as the complexity of the circuits are the disadvantages of this method. Also, another question is: where 
and how many breakpoints should be selected. Although this problem has been examined in detail for sine function synthesis [19], there is no strategy for breakpoint selection of EXP function.

The third group deals with presenting new approximations of function in a limited range [14], [15], [20], [21] in which each term of the approximated series is realized using a voltage-mode [14], [15] or current-mode [20] circuits. The method presented in [14], [15] use pseudo-exponential functions which result in simple realization of the circuits and relatively low power consumption, however the precision and dynamic range have been sacrificed. Higher-order terms proposed in [20] benefit from higher dynamic range in comparison with above mentioned methods, at the cost of complex implemented circuits and lack of area efficiency which leads to increasing in power dissipation. Beside these classifications, designing the circuit with single power supply is preferred to those in dual mode; while the circuits reported in [7], [10], [13-15], [18], [19] require dual supply voltages. In conclusion, a circuit with wide dynamic range, high accuracy and low power consumption is expected as a further work. We introduced a method in [21] to satisfy these properties as well.

This paper proposes a new approximation for exponential function which significantly extends the dB-linear output dynamic range of the EXPFG circuit. The new function is obtained by using linear least squares approach to allocate optimum coefficient of a new fitted function on the EXP function. The resulted topology provides a $78 \mathrm{~dB}$ output range with the linearity error as low as $\pm 0.5 \mathrm{~dB}$, which demonstrates a significant improvement in comparison with the previously reported works. Unlike to the some reported circuits which were designed in the subthreshold region, the proposed configuration operates in the saturation region, thus provides acceptable bandwidth for the circuit. In addition, Monte Carlo analysis is performed to verify the robustness of the circuit against the process variation. Also, HSPICE and Cadence simulators are utilized to validate the theoretical formulations. The paper is organized in 6 sections: The proposed approach for implementation of EXP function is presented in Sec. 2, followed by the transistor level design of the proposed approximation in Sec. 3. In Sec. 4, post layout simulation results are presented to prove the efficiency of the design. The performance analysis of the circuit is presented in Sec. 5, and finally, conclusions are outlined in Sec. 6.

\section{Proposed Approach}

The proposed approach begins with presenting a new approximation formula for the exponential function as:

$$
\mathrm{e}^{x} \approx a x^{4}+b x^{2}+c x+d
$$

where $a, b, c$ and $d$ are the coefficients and $x$ is the independent variable. In order to avoid complex circuitry configuration, specific terms including $x^{4}, x^{2}$ and $x$ as well as a constant value of $d$ are only considered. In other words, the main difference in the proposed approximation rather than conventional approximations presented in [14], [18], [21] is simpler and more accurate realization in the CMOS technology. Moreover, in comparison with these works the input range of the resulted function is wider. The reason for choosing the above mentioned terms is that the term $x^{2}$ can be readily realized using a squarer (SQ) circuit, and two cascaded SQ circuits are used to provide the term $x^{4}$. Also the term $x$ is directly generated through the input with proper gain as a coefficient.

It should be pointed out that in the exponential function circuits, there is a tradeoff between the accuracy and the input/output dynamic range; a narrow dynamic range results in higher precision, while a wide dynamic range usually decreases the accuracy of the circuit.

The objective of this paper is presenting a method to design a circuit which provides high precision performance as well as the wide input/output dynamic range. The proposed idea can be briefly explained as follows:

In order to increase the precision of EXP circuit, the input range (amplitude of the input signal) of the circuit is halved. In the next phase, the decreased signal range is compensated by squaring output signal. This means that in the first phase a high accurate circuit is implemented, and in the second, wide output range is expected. The overall procedure can be explained by the following equation:

$$
\mathrm{e}^{x}=\left(\mathrm{e}^{0.5 x}\right)^{2}
$$

In the view of circuit realization, the structure consists of two stages; function of $\mathrm{e}^{0.5 x}$ is realized in the first stage, then the resulted output is applied to the SQ circuit such that the final circuit implements function of $\mathrm{e}^{x}$.

The coefficients of polynomial in (1) are calculated using linear least squares optimization method which found as: $a=0.01471, b=-0.3623, c=2.306$ and $d=0.1118$. In this regard, MATLAB curve fitting toolbox is employed. In the first step, the desired interval for the fitting is defined in the command window which is [0 9]. Secondly, the function for the mentioned interval is defined. In the next step, custom type of polynomials including coefficients and terms are defined which are $a, b, c$ and $d$ for coefficients and

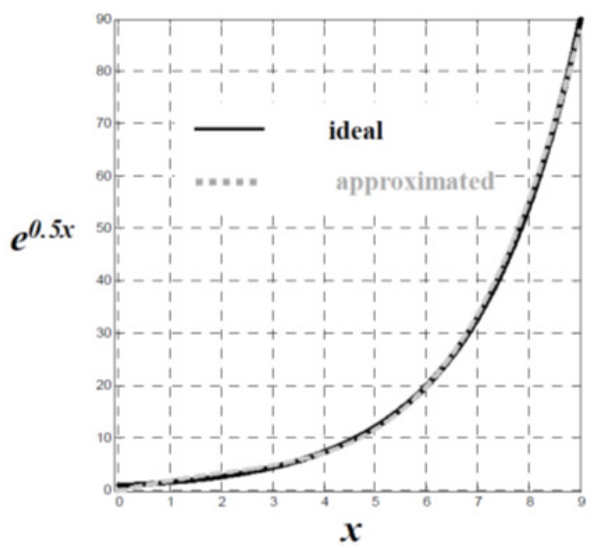

Fig. 1. Approximated EXP function using linear least squares method. 
and $x^{4}, x^{2}, x$ for terms. In the next step method of fitting is chosen which is linear least squares approach. Finally, the polynomial of $a x^{4}+b x^{2}+c x+d$ is fitted on the exponential function in the defined interval and the optimized coefficients as well as the RMSE error are given. Therefore the final approximation formula for function of $\mathrm{e}^{0.5 x}$ is given by:

$$
\mathrm{e}^{0.5 x}=0.01471 x^{4}-0.3623 x^{2}+2.306 x+0.1118
$$

where $0<x<9$. In order to simple implementation of the above approximation, considering circuitry conditions, the following formula is derived:

$$
\mathrm{e}^{0.5 x}=(0.348 x)^{4}-(0.602 x)^{2}+2.306 x+0.1118 .
$$

The approximated curve (dashed line) and ideal exponential function (solid line) are shown in Fig. 1.

\section{Circuit Description}

The design procedure of the circuit begins with the selection of bias current of $10 \mu \mathrm{A}$ as a normalized current. The main building block which is employed in the EXPFG circuit is current squaring circuit shown in Fig. 2(a). Consider a loop of MOS transistor $M 1$ to $M 4$; writing KVL in the gate-source voltages of these transistors yields:

$$
V_{\mathrm{GS} 1}+V_{\mathrm{GS} 2}=V_{\mathrm{GS} 3}+V_{\mathrm{GS} 4} .
$$

Since all transistors are biased in saturation region, then $V_{\mathrm{GS}}=V_{\mathrm{t}}+\sqrt{\frac{I_{\mathrm{D}}}{K}}$; Replacing in (5) and assuming that transconductance parameter and threshold voltage of all transistors are well matched, we have:

$$
\sqrt{I_{\mathrm{DS} 1}}+\sqrt{I_{\mathrm{DS} 2}}=\sqrt{I_{\mathrm{DS} 3}}+\sqrt{I_{\mathrm{DS} 4}} \text {. }
$$

Deriving currents of $I_{\mathrm{DS} 3}$ and $I_{\mathrm{DS} 4}$ by KCL at nodes A and B:

$$
\begin{gathered}
I_{\mathrm{DS} 3}=I_{\mathrm{SQ}}+I_{\mathrm{in}}, \\
I_{\mathrm{DS} 4}=I_{\mathrm{SQ}}-I_{\text {in }}
\end{gathered}
$$

where $I_{\text {in }}$ and $I_{\mathrm{SQ}}$ represent input and squaring currents, respectively.

Since $I_{\mathrm{DS} 1}=I_{\mathrm{DS} 2}=I_{\mathrm{B}}, \quad\left(I_{\mathrm{B}}\right.$ is the bias current $)$ substituting (7) and (8) into (6) and then squaring both sides twice, the output of the circuit can be derived as:

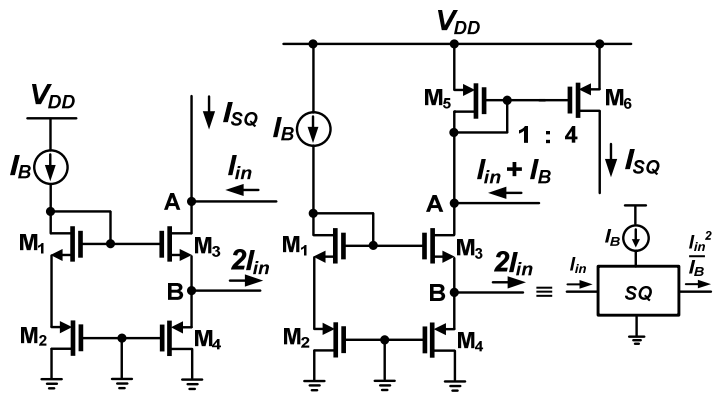

(a)

(b)

Fig. 2. Current-mode SQ circuit: (a) presented in [22], (b) modified circuit.

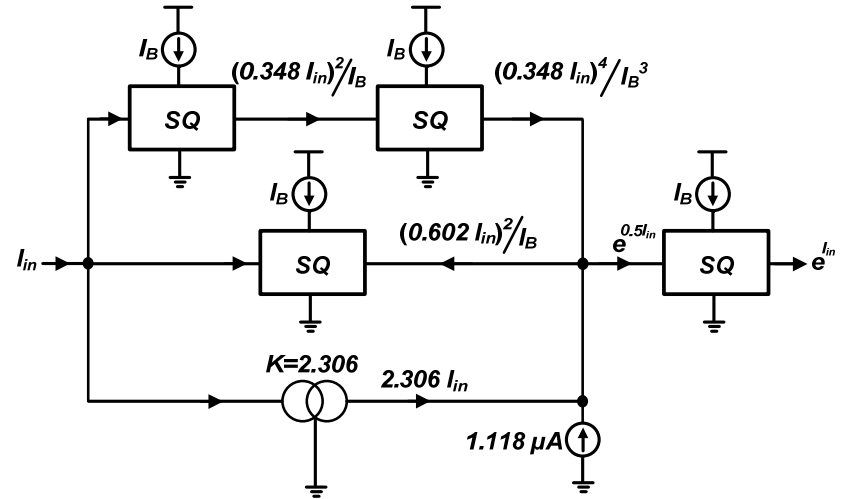

Fig. 3. The proposed block diagram for implementation of EXPFG.

$$
I_{\mathrm{SQ}}=\frac{I_{\text {in }}^{2}}{4 I_{\mathrm{B}}}+I_{\mathrm{B}} .
$$

It can be clearly seen that the squaring of input current is obtained at the output. Also, the bias current of $I_{\mathrm{B}}$ is considered as a normalized current.

In order to simple using of SQ circuit, it is better to consider the output current as $I_{\text {in }}{ }^{2} / I_{\mathrm{B}}$. Therefore, the modified circuit of Fig. 2(b) is presented to provide the required function. This can be readily done by applying $I_{\mathrm{DS} 3}=I_{\mathrm{SQ}} / 4$ $+I_{\text {in }}+I_{\mathrm{B}}$ and $I_{\mathrm{DS} 4}=I_{\mathrm{SQ}} / 4-I_{\text {in }}+I_{\mathrm{B}}$. Following the above procedure, the desired output is obtained.

The proposed block diagram for implementation of EXPFG circuit is shown in Fig. 3. The term of $(0.348 x)^{4}$ is implemented in the upper branch by cascading two SQ circuits in the circuitry form of $\left(0.348 I_{\text {in }}\right)^{4} / I_{\mathrm{B}}{ }^{3}$. The middle branch realizes $(0.602 x)^{2}$ in the form of $\left(0.602 I_{\text {in }}\right)^{2} / I_{\mathrm{B}}$ using single SQ circuit. The next branch realizes linear term of approximation which composed of a current mirror with the gain of 2.306, and finally, the DC current source implements the constant term of $1.118 \mu \mathrm{A}$. Take notice that since the circuit is designed in the current mode, current mirrors with defined gains are employed to realize coefficients of (4), ( $a, \mathrm{~b}, \mathrm{c}$ and $d)$.

\section{Post Layout Simulation Results}

In this section, HSPICE and Cadence simulators are utilized to prove the performance of the circuit for $0.18 \mu \mathrm{m}$ CMOS technology. The layout pattern of the EXPFG circuit drawn by Cadence is depicted in Fig. 4 which verifies that the circuit can be realized in $75 \mu \mathrm{m} \times 43 \mu \mathrm{m}$ active area. Figures 5 and 6 demonstrate HSPICE results of the circuit in the exponential form $\left(\mathrm{e}^{0.5 I_{\text {in }}}\right)$ and linear mode, respectively, which shows an output range of $38 \mathrm{~dB}$. It is compared with the ideal output which is confirmed by simulation results obtained by HSPICE. The error measurement is shown to validate the precision of the circuit in which the average of error is $0.16 \mathrm{~dB}$. As discussed, in order to have higher accuracy, in the second phase by squaring $\mathrm{e}^{0.5 I_{\text {in }}}$ the full exponential function $\left(\mathrm{e}^{I_{\text {in }}}\right)$ is generated. Figure 7 shows the exponential output of the circuit in the form of $\mathrm{e}^{I_{\text {in }}}$ with $78 \mathrm{~dB}$ output dynamic range. In addition, 


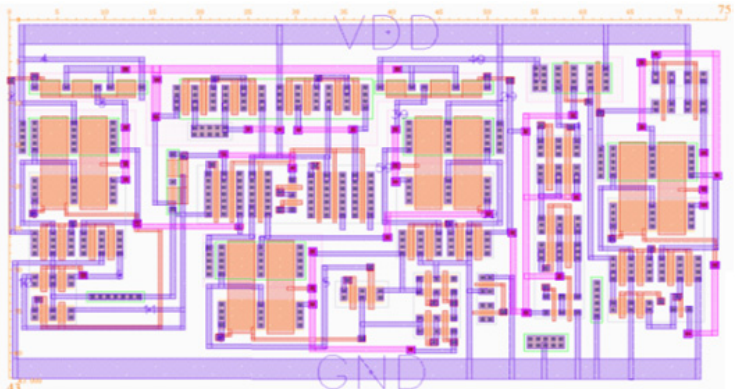

Fig. 4. Layout pattern of the proposed EXPFG circuit.

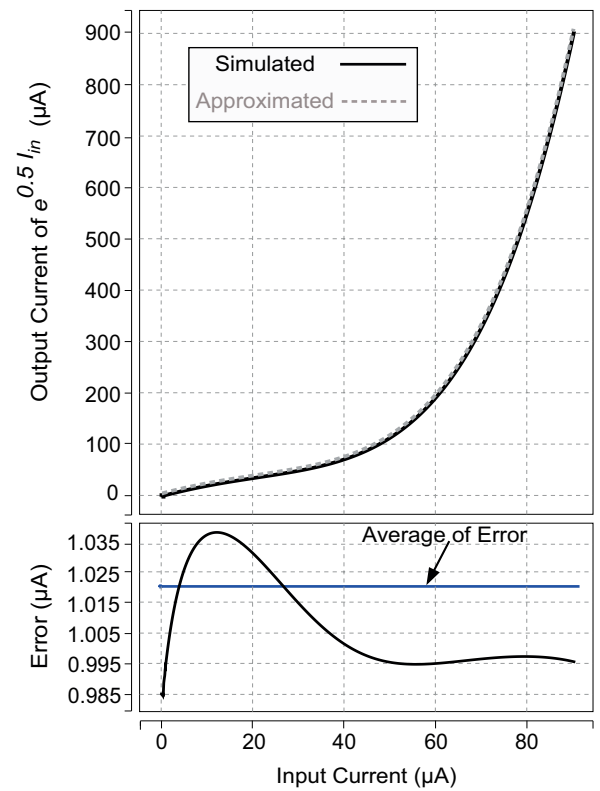

Fig. 5. HSPICE simulation result for $\mathrm{e}^{0.5 I_{\text {in }}}$ function.

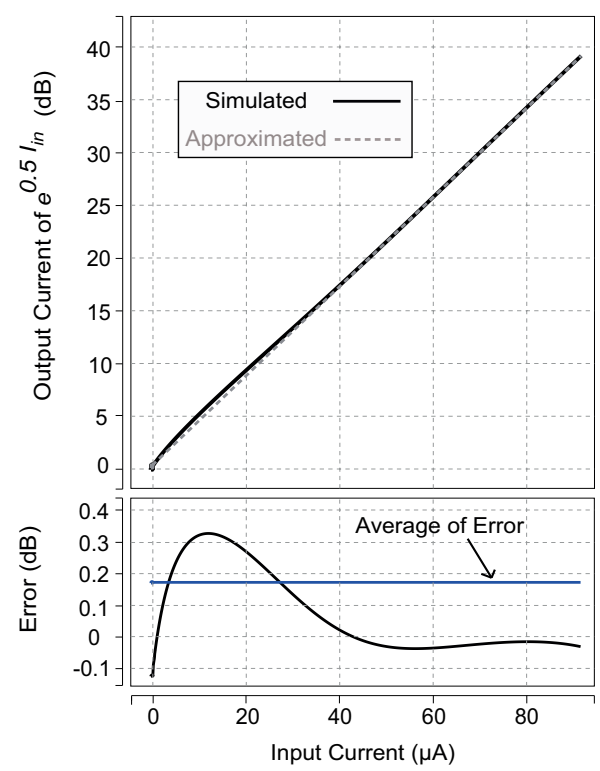

Fig. 6. HSPICE result of $\mathrm{e}^{0.5 I_{\text {in }}}$ in $\mathrm{dB}$ scale.

the average error of $0.29 \mathrm{~dB}$ shows a highly accurate performance of the circuit.

To prove the efficiency of the circuit, frequency response is provided. Since some reported works work in subthreshold region [10-13], and some others have complex structure [11], [15], [21], thus their bandwidth is limited, while in this work all of the transistors are biased in the saturation region. Frequency response of the EXPFG circuit in Fig. 9 demonstrates that $-3 \mathrm{~dB}$ bandwidth is $67 \mathrm{MHz}$. In order to calculate $-3 \mathrm{~dB}$ bandwidth of the circuit, the input

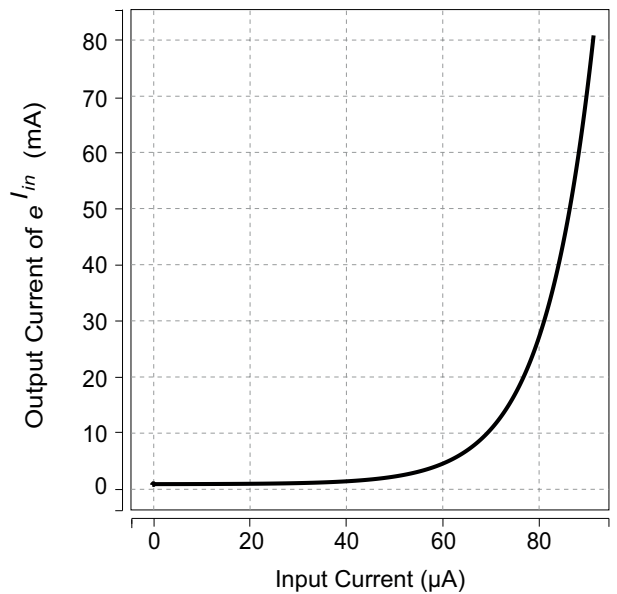

Fig. 7. HSPICE simulation result for $\mathrm{e}^{\mathrm{I}_{\text {in }}}$ function.

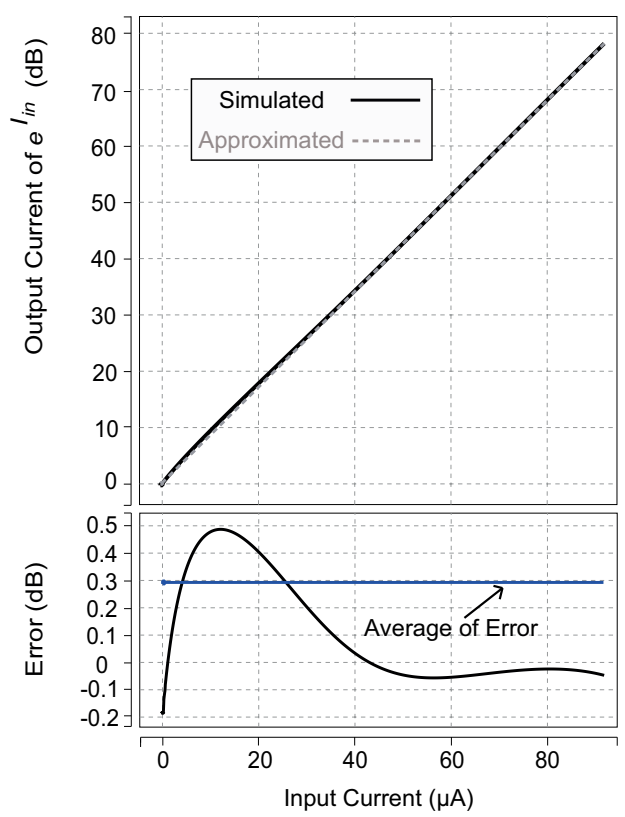

Fig. 8. HSPICE simulation result of $\mathrm{e}^{I_{\text {in }}}$ in $\mathrm{dB}$ scale and its error measurement.

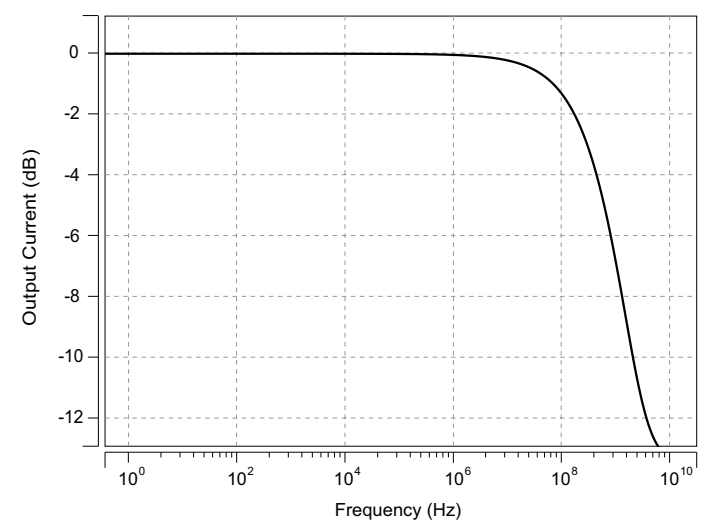

Fig. 9. Frequency response of the EXPFG circuit. 


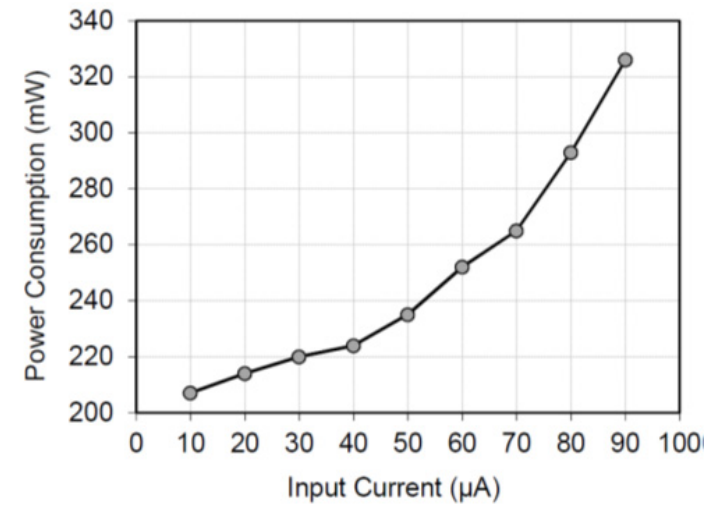

Fig. 10. Power dissipation of the proposed circuit versus different input currents.

signal is set to the value that the output current be $1 \mathrm{~dB}$, and then the frequency in which the gain is $-3 \mathrm{~dB}$ is calculated.

Figure 10 illustrates the power consumption of the EXPFG circuit for different input currents. As expected, the power dissipation is increased when the input current increased. The maximum power consumption is obtained $0.326 \mathrm{~mW}$ with the input current of $90 \mu \mathrm{A}$, while the average of power consumption is found as low as $0.248 \mathrm{~mW}$.

\section{Error Analysis in the Circuits}

The error analysis of EXPFG circuit is examined in this section. Considering (1), the main error sources originate from terms of $x^{2}$ and $x^{4}$, which are implemented by SQ and two cascaded SQ circuits, respectively. As discussed, SQ circuit is designed using translinear loop, which was considered in an ideal form, and then (5) was derived, while the body effect was ignored. In a MOS transistor body effect is given by:

$$
V_{\mathrm{t}}=V_{\mathrm{t} 0}+\gamma\left[\sqrt{\left(2 \phi_{\mathrm{b}}+\left|V_{\mathrm{SB}}\right|\right)}-\sqrt{2 \phi_{\mathrm{b}}}\right] .
$$

Since bulk of transistors $\mathrm{M}_{2}$ and $\mathrm{M}_{4}$ are connected to the source terminal, therefore $V_{\mathrm{SB}}=0$ and then $V_{\mathrm{t}}=V_{\mathrm{t} 0}$, while in $\mathrm{M}_{1}$ and $\mathrm{M}_{3}$ transistors $V_{\mathrm{SB}} \neq 0$. Supposing the probable mismatch for transistors $\mathrm{M}_{1}$ and $\mathrm{M}_{3}$ as:

$$
\begin{aligned}
& V_{\mathrm{GS} 1}=V_{\mathrm{t} 1}+\Delta V_{1}, \\
& V_{\mathrm{GS} 3}=V_{\mathrm{t} 3}+\Delta V_{3} .
\end{aligned}
$$

Substituting (11) and (12) into (5) yields:

$$
V_{\mathrm{t} 1}+\Delta V_{1_{1}}+V_{\mathrm{GS} 2}=V_{\mathrm{t} 3}+\Delta V_{3}+V_{\mathrm{GS} 4}
$$

where $V_{\mathrm{t} 1}=V_{\mathrm{t}}+\delta, V_{\mathrm{t} 3}=V_{\mathrm{t}}-\delta$ and $\delta$ is the mismatch value among $V_{\mathrm{t} 1}$ and $V_{\mathrm{t} 3}$.

Considering $I_{\mathrm{B}}=K \Delta V^{2}$ then by some algebra, the output current can be written as:

$$
I_{\text {out }}^{\prime}=\frac{I_{\text {in }}^{2}}{4 K \Delta V(\Delta V+2 \delta)}+\frac{K \Delta V(\Delta V+4 \delta)}{1+\frac{2 \delta}{\Delta V}} .
$$

Subtracting (14) from (9), and ignoring high-order terms containing $\Delta V^{n}(n=3,4,5)$ and also $\delta^{2}$, error of the SQ circuit is derived:

$$
\left|I_{\text {error }}^{\prime}\right|_{2}=\frac{\delta}{2 K \Delta V^{2}} I_{\text {in }}^{2}+4 K \delta \Delta V^{2} .
$$

where $\left|I_{\text {error }}^{\prime}\right|_{2}$ is the error of SQ circuit. The same analysis is simply carried out for two cascaded SQ circuits which results in the error as:

$\left|I_{\text {out }}^{\prime}\right|_{4}=\frac{I_{\text {in }}{ }^{4}(1+2 \delta)+8 \delta I_{\mathrm{B}}{ }^{2} I_{\text {in }}{ }^{2}}{I_{\mathrm{B}}{ }^{3}\left(1+2 \delta+\frac{4 \delta^{2}}{\Delta V^{2}}\right)}+\frac{4 K \delta \Delta V-\frac{2 \delta I_{\mathrm{B}}}{\Delta V}}{1+\frac{2 \delta}{\Delta V}}$,

$\left|I_{\text {error }}^{\prime}\right|_{4}=\frac{\frac{4 \delta^{2}}{\Delta V^{2}}\left(I_{\text {in }}{ }^{4}\right)-8 \delta I_{\mathrm{B}}{ }^{2} I_{\text {in }}{ }^{2}}{I_{\mathrm{B}}{ }^{3}\left(1+2 \delta+\frac{4 \delta^{2}}{\Delta V^{2}}\right)}-\frac{4 K \delta \Delta V-\frac{2 \delta I_{\mathrm{B}}}{\Delta V}}{1+\frac{2 \delta}{\Delta V}}$

where $\mid I^{\prime}$ error $\mid 4$ is the error of two cascaded SQ circuits. Considering (15) and (17), the mismatch error for threshold voltages can be neglected as long as:

$$
\Delta V \gg 2 \delta, \Delta V \gg 4 \delta, 1 \gg \frac{2 \delta}{\Delta V} .
$$

Replacing (15) and (17) into (3), the error quantity for EXPFG circuit is computed as follows:

$$
I_{\text {error }}^{\prime}=0.0147\left(\left|I_{\text {error }}^{\prime}\right|_{4}\right)-0.3623\left(\left|I_{\text {error }}^{\prime}\right|_{2}\right) .
$$

This equation implies that the error of two individual blocks are subtracted, thus the error at the output of EXPFG circuit is smaller than the errors in each SQ and two cascaded SQ circuits. Also, the coefficients of errors are less than $1(0.0147$ and 0.3623$)$. This means that each resulted error is reduced which in turn degrades the overall error of the circuit. In order to verify this analysis and also to evaluate circuit robustness against the process variation, the Monte Carlo statistical analysis is carried out taking $\pm 5 \%$ Gaussian distribution in the variation of transistors threshold voltage. The simulation results are shown in Fig. 11 where $64 \%$ of the occurrences lead to the error less than $\pm 1 \%$, while $13 \%$ of samples occurred with the error more than $\pm 2 \%$. Take notice that to calculate the error, output current (not ideal output) is compared with those obtained by Monte Carlo analysis. To prove the high linearity of the proposed circuit in $\mathrm{dB}$ against the process tolerances, the same analysis with the similar conditions of previous simulation is performed. The simulation results are shown in Fig. 12, and demonstrate that $84 \%$ of the samples have the error of less than $\pm 0.6 \mathrm{~dB}$, and just $6 \%$ of the occurrences lead to the error of more than $\pm 1 \mathrm{~dB}$. Also, the mismatch of the transconductance parameter can be modeled as follows:

$$
\begin{aligned}
& K_{\mathrm{P}}=K+\Delta k K, \\
& K_{\mathrm{N}}=K-\Delta k K
\end{aligned}
$$

where $K$ is a mean value and $\Delta k$ is a mismatch percentage of the parameter. Assuming this, (6) becomes: 


$$
\sqrt{\frac{I_{\mathrm{B}}}{K+\Delta k K}}+\sqrt{\frac{I_{\mathrm{B}}}{K-\Delta k K}}=\sqrt{\frac{I_{\text {out }}^{\prime \prime}+I_{\text {in }}}{K+\Delta k K}}+\sqrt{\frac{I_{\text {out }}^{\prime \prime}-I_{\text {in }}}{K-\Delta k K}}
$$

where $I^{\prime \prime}$ out is the output of the squaring circuit. Simplifying (21) and ignoring terms containing $\Delta k^{2}$ (because $\Delta k<<1$ ), output current of squarer circuit can be written as:

$$
I_{\text {out }}^{\prime \prime} \approx \frac{I_{\text {in }}^{2}+\Delta k I_{\text {in }}}{4 I_{\mathrm{B}}+2 \Delta k I_{B} I_{\text {in }}} .
$$

Subtracting (22) from (9), following the same procedure of above mentioned mismatch for threshold voltage, then by some algebra one can find the error quantity for SQ, cascaded SQ and EXPFG circuits as follow:

$$
\begin{gathered}
\left|I_{\text {error }}^{\prime \prime}\right|_{2}=\frac{I_{\text {in }}{ }^{2}+4 \Delta k I_{\mathrm{B}} I_{\text {in }}}{I_{\mathrm{B}}+2 \Delta k I_{\text {in }}}, \\
\left|I_{\text {error }}^{\prime \prime}\right|_{4}=\frac{2 \Delta k I_{\mathrm{B}} I_{\text {in }}{ }^{4}-\Delta k^{2} I_{\mathrm{B}}{ }^{2} I_{\text {in }}{ }^{2}-4 \Delta k I_{\mathrm{B}}}{1+2 I_{\mathrm{B}}{ }^{2}+4 I_{\mathrm{B}} I_{\text {in }} \Delta k-I_{\text {in }} \Delta k^{2}-I_{\mathrm{B}} I_{\text {in }} \Delta k^{4}} .
\end{gathered}
$$

Replacing (23) and (24) into (3), the error quantity for EXPFG circuit is computed as:

$$
I_{\text {error }}^{\prime \prime}=0.026\left(\left|I^{\prime \prime}{ }_{\text {error }}\right|_{4}\right)+0.147\left(\left|I^{\prime \prime}{ }_{\text {error }}\right|_{2}\right) \text {. }
$$

Considering that the coefficients in (25) as well as $\left|I_{\text {error }}\right|_{4}$ and $\mid I_{\text {error }}^{\prime}$ are less than one, we can ignore the mismatch of transconductance parameters. The Monte Carlo analysis is also carried out for this parameter in variation of aspect ratio of the transistors (considering $\left.K=0.5 \mu_{0} C_{\mathrm{OX}}(W / L)\right)$. The result is shown in Fig. 13. For channel length modulation effect, it is well known that, for long channel MOS transistors, the perfect square-law equation agrees with experimental results to a great extent [24], and since channel length for the core circuit (squaring circuit) is $3.8 \mu \mathrm{m}$, therefore, this effect can be negligible.

Another factor which affects the circuit performance is temperature variation which originates from dependency of the threshold voltage in CMOS circuits to the temperature. Considering the threshold voltage of MOS transistor as:

$$
V_{\mathrm{T}}=\phi_{\mathrm{ms}}-\frac{Q_{\mathrm{ss}}}{C_{\mathrm{ox}}}+2 \phi_{\mathrm{F}}+\gamma \sqrt{2 \phi_{\mathrm{F}}}
$$

where $\phi_{\mathrm{ms}}=\phi_{\mathrm{T}} \ln \left(N_{\mathrm{A}} N_{\mathrm{G}} / n_{\mathrm{i}}^{2}\right)$ is the contact potential difference between gate and substrate, $N_{\mathrm{G}}$ and $N_{\mathrm{A}}$ are the gate and substrate doping levels, respectively and $n_{\mathrm{i}}$ is the intrinsic electron-carrier concentration of $\mathrm{Si}, Q_{\mathrm{ss}}$ the surfacestate charge density per unit area, and $C_{\text {ox }}$ the oxide capacitance; $\gamma$ is a body effect parameter and $\phi_{\mathrm{F}}=\phi_{\mathrm{T}} \ln \left(N_{\mathrm{A}} / n_{\mathrm{i}}\right)$ is the Fermi potential of substrate with the thermal voltage of $\phi_{\mathrm{T}}=k T / q$. In the above equation, both of $\phi_{\mathrm{ms}}$ and $\phi_{\mathrm{F}}$ depends on $\phi_{\mathrm{T}}$, and are only parameters which depend on threshold voltage $\left(\partial V_{\mathrm{T}} / \partial T\right)$ which can be written as [23]:

$$
\frac{\partial V_{\mathrm{T}}}{\partial T}=\frac{\partial \phi_{\mathrm{ms}}}{\partial T}+2 \frac{\partial \phi_{\mathrm{F}}}{\partial T}+\frac{\gamma}{\sqrt{2 \phi_{\mathrm{F}}}} \frac{\partial \phi_{\mathrm{F}}}{\partial T}
$$

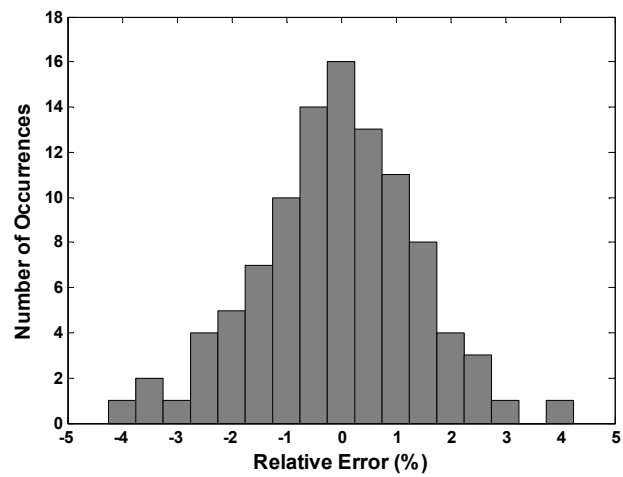

Fig. 11. Monte Carlo analysis of EXP circuit for relative error.

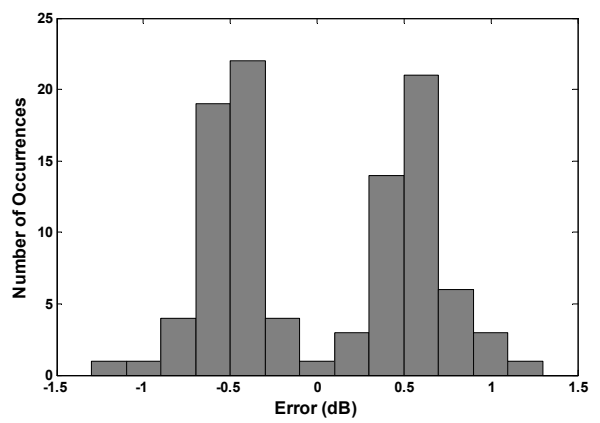

Fig. 12. The result of Monte Carlo analysis against the process variation to prove the linearity of the circuit.

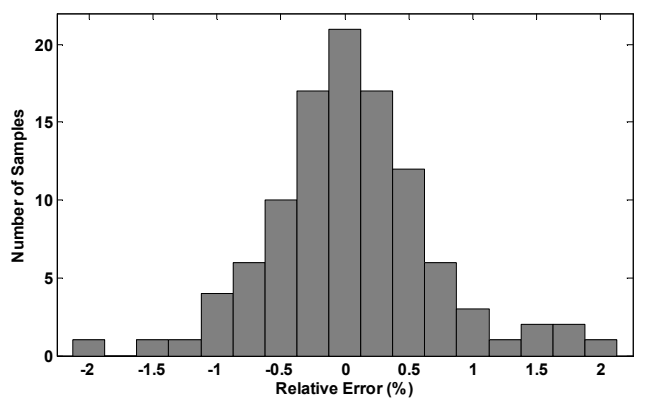

Fig. 13. Monte Carlo analysis for mismatch in transconductance parameters in EXPFG circuit.

In order to examine the performance of EXPFG circuit versus temperature tolerances, SQ circuit which is the main block in EXPFG as well as the current mirrors are discussed separately: In the current mirror circuits, corresponding transistors carry equal drain-to-source currents because of equal voltages of $V_{\mathrm{GS}}$ and $V_{\mathrm{DS}}$. Since these voltages of corresponding transistors are equal in all conditions, any variations in threshold voltage are automatically compensated. Thus, the performance of the circuit does not depend for the probable temperature tolerances. Regarding SQ circuit, the output formula demonstrates dependency to the threshold voltage. As we discussed, the circuit is based on the translinear principle which obtains from a loop of gate-to-source voltages. Considering (15) and (17), this voltage is affected by threshold voltage and consequently by temperature variation. One can see the effect of threshold voltage at the output current by $\delta$ parameter:

$$
I_{\text {out }}^{\prime}=\frac{I_{\text {in }}{ }^{2}}{4 K \Delta V(\Delta V+2 \delta)}+\frac{K \Delta V(\Delta V+4 \delta)}{1+\frac{2 \delta}{\Delta V}} .
$$


Considering (28), $\delta$ is very small in comparison with constant voltage of $\Delta V$ which is proportion of bias current. Therefore, it is negligible in the terms of $(\Delta V+2 \delta)$ and $(\Delta V+4 \delta)$. Accordingly, in the term of $(1+2 \delta / \Delta V), \delta$ is divided to $\Delta V$ which is very large. On the other hand, the concluded value is added to 1 .

As a result, threshold voltage tolerances caused by temperature variation do not notably influence the performance of the main building block e.g. SQ circuit. For the complete circuit the simulation is carried out and shown in Fig. 13, where the relative error of the circuit versus temperature validates the robustness of the EXPFG circuit against temperature variations. In this simulation, output of EXPFG at $20^{\circ} \mathrm{C}$ is supposed as the non-error output, and then the circuit is simulated in different temperatures. The results show that the maximum error occurred at $-40^{\circ} \mathrm{C}$ with $0.77 \%$ error.

For channel length modulation effect, it is well known that, for long channel MOS transistors, the perfect squarelaw equation agrees with experimental results to a great extent [24], and since channel length for the core circuit (squaring circuit) is $3.8 \mu \mathrm{m}$, therefore, this effect can be negligible. In order to show the applicability of the proposed EXP circuit, it is used to realize a dB-linear variablegain amplifier (VGA) shown in Fig. 15, an important application in radio engineering field [25], [26]. The proposed VGA employs the exponential characteristic realized in Fig. 3 and also a multiplier/divider which is implemented based on the circuit of Fig. 1 using square-difference algebraic identity: $(x+y)^{2}-(x-y)^{2}=4 x y$. Therefore using two squaring circuits and one subtractor, the desired output using (9) is derived as:

$$
I_{\text {out }(\mathrm{VGA})}=I_{\text {out2(VGA) }}-I_{\text {out } 1(\mathrm{VGA})}=\frac{I_{\text {out(EXP) }}}{I_{\mathrm{B}}} I_{\mathrm{in}(\mathrm{VGA})}
$$

where $I_{\mathrm{B}}$ is the reference current, $I_{\text {out(EXP) }}$ represents the output current of EXP circuit, having the output current explained in Fig. $3\left(I_{\mathrm{B}} \exp \left(I_{\text {in }} / I_{\mathrm{B}}\right)\right)$, while $I_{\text {out(VGA) }}$ expresses the output current of the dB-linear VGA circuit. Then, by replacing $I_{\mathrm{B}} \exp \left(I_{\mathrm{in}} / I_{\mathrm{B}}\right)$ in (29) the output is given by:

$$
I_{\mathrm{out}(\mathrm{VGA})}=\exp \left(\frac{I_{\mathrm{in}}}{I_{\mathrm{B}}}\right) I_{\mathrm{in}(\mathrm{VGA})}=G I_{\mathrm{in}(\mathrm{VGA})} .
$$

Thus, the gain $G$ of VGA circuit can be exponentially tuned by current of $I_{\text {in }}$ through the EXP circuit.

Table 1 summarizes the related results of EXPFG circuit and allows a deeper comparison. Ref. [10] benefits from high $\mathrm{dB}$ gain, but it needs double power supply, and also its bandwidth and output range are limited to $0.105 \mathrm{MHz}$ and $\pm 48 \mathrm{~dB}$ respectively, while our proposed circuit range is 0 to $78 \mathrm{~dB}$. Note that Refs. [7] and [10] have been designed in weak inversion, thus their power consumption is low. As seen their bandwidth is narrow, and also they use double power supply. Ref. [15] benefits from lower power dissipation and acceptable precision, but they suffer from low bandwidth and poor output dynamic

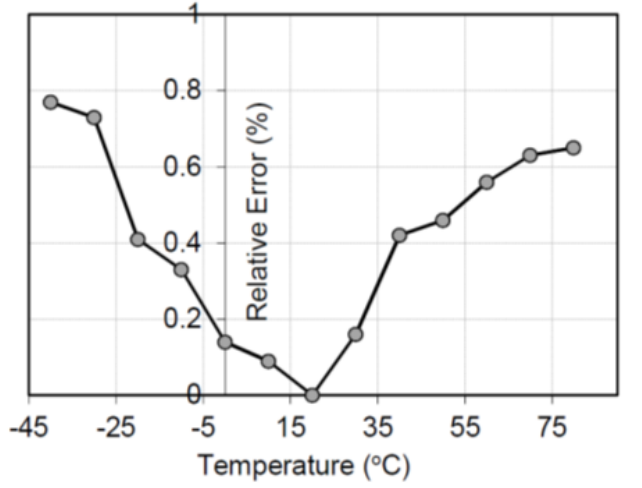

Fig. 14. Relative error of EXPFG circuit versus different temperatures.

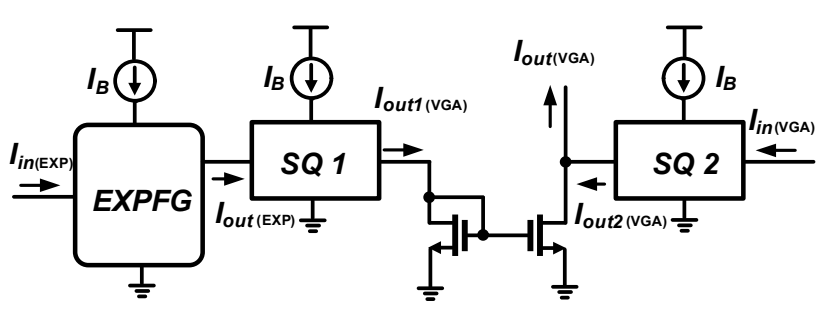

Fig. 15. VGA implementation using designed EXP circuit.

\begin{tabular}{|c|c|c|c|c|c|c|}
\hline Ref. & $\begin{array}{c}\text { Gain } \\
(\mathrm{dB})\end{array}$ & $\begin{array}{c}\text { Accuracy } \\
(\text { Error in } \\
\mathrm{dB})\end{array}$ & $\begin{array}{c}\text { BW } \\
(\mathrm{MHz})\end{array}$ & $\begin{array}{c}\text { Supply } \\
(\mathrm{V})\end{array}$ & $\begin{array}{c}\text { Power } \\
(\mathrm{mW})\end{array}$ & $\begin{array}{c}\text { Tech. } \\
(\mu \mathrm{m})\end{array}$ \\
\hline$[7]$ & 71 & 0.3 & 0.181 & \pm 0.75 & 0.013 & 0.35 \\
\hline$[10]$ & 96 & \pm 0.5 & 0.105 & \pm 0.75 & 0.0063 & 0.35 \\
\hline$[11]$ & 48 & \pm 0.5 & 3 & 1.8 & 0.549 & 0.18 \\
\hline$[12]$ & 40 & \pm 0.75 & $\mathrm{NA}$ & 1.5 & 0.6 & 0.35 \\
\hline$[15]$ & 66 & \pm 0.5 & 31 & \pm 0.75 & 0.17 & 0.18 \\
\hline$[16]$ & 41 & \pm 1 & 168.8 & 2.5 & 0.535 & 0.13 \\
\hline$[20]$ & 70 & \pm 1 & 32 & 1 & 0.08 & 0.18 \\
\hline Proposed & 78 & $\leq \pm 0.5$ & 67 & 1.5 & 0.326 & 0.18 \\
\hline
\end{tabular}

Tab. 1. Comparative parameters of the proposed circuit with other recent works.

range. Ref. [21] has a good performance in output dynamic range and power consumption, but its accuracy is comparatively low. In fact, we sacrificed the power consumption by implementing higher-order terms at the cost of more accuracy. In addition, its bandwidth is as low as one-half of the proposed circuit.

\section{Conclusion}

In this paper, a new CMOS exponential circuit has been presented. The proposed circuit enjoyed these attractive features: 1) Presenting a new approximation for exponential function in the defined range. 2) Extending output dynamic range by proposing cascaded stages up to $78 \mathrm{~dB}$. 3) Obtaining very accurate performance with average error less than $0.3 \mathrm{~dB}$. 4) Achieving satisfactory bandwidth resulted from current-mode realization which provided simple and intuitive configuration 5) Demonstrating acceptable performance against the process variation. 


\section{References}

[1] MATEO, J., SÁNCHEZ-MORLA, E., SANTOS, J. L. A new method for removal of powerline interference in ECG and EEG recordings. Computers and Electrical Engineering, 2015, vol. 45, p. 235-48. DOI: 10.1016/j.compeleceng.2014.12.006

[2] VASUNDHARA, PUHAN. N. B., PANDA, G. De-correlated improved adaptive exponential FLAF-based nonlinear adaptive feedback cancellation for hearing aids. IEEE Transactions on Circuits and Systems I: Regular Papers, 2017, vol. 65, no. 2, p. 650-662. DOI: 10.1109/TCSI.2017.2730235

[3] DUONG, Q. H., LE, Q., KIM, C. W., et al. A 95-dB linear lowpower variable gain amplifier. IEEE Transactions on Circuits and Systems I: Regular Papers, 2006, vol. 53, no. 8, p. 1648-1657. DOI: 10.1109/TCSI.2006.879058

[4] NADERI, S. A., OZOGUZ, S. Design of high-linear, highprecision analog multiplier free from body effect. Turkish Journal of Electrical Engineering and Computer Sciences, 2016, vol. 24, p. 820-832. DOI:10.3906/elk-1307-159

[5] NADERI, S. A. High-precision CMOS analog computational circuits based on a new linearly tunable OTA. Radioengineering, 2016, vol. 25, no. 2, p. 297-304. DOI: 10.13164/re.2016.0297

[6] AL-SUHAIBANI, E., AL-ABSI, M. A. A new CMOS currentmode controllable-gain square rooting circuit using MOSFET in subthreshold. Analog Integrated Circuits and Signal Processing, 2015, vol. 82, no. 2, p. 431-434. DOI 10.1007/s10470-015-0488-0

[7] AL-TAMIMI, K. M., AL-ABSI, M. A., ABUELMA'ATTI, M. T. Temperature insensitive current-mode CMOS exponential function generator and its application in variable gain amplifier. Microelectronics Journal, 2014, vol. 45, no. 3, p. 345-354. DOI: 10.1016/j.mejo.2013.12.010

[8] WEY, T., JEMISON, W. An automatic gain control circuit with $\mathrm{TiO}_{2}$ memristor variable gain amplifier. Analog Integrated Circuits and Signal Processing, 2012, vol. 73, no. 3, p. 663-672. DOI: $10.1007 / \mathrm{s} 10470-012-9860-5$

[9] POPA, C. High-accuracy function synthesizer circuit with applications in signal processing. EURASIP Journal on Advances in Signal Processing, 2012, vol. 146, p. 1-11. DOI: 10.1186/1687-6180-2012146

[10] AL-TAMIMI, K. M., AL-ABSI, M. A. A $6.13 \mu \mathrm{W}$ and $96 \mathrm{~dB}$ CMOS exponential generator. IEEE Transactions on Very Large Scale Integration (VLSI) Systems, 2014, vol. 22, no. 11, p. 2443-2447. DOI: 10.1109/TVLSI.2013.2292093

[11] YANG, S., WANG, C. A low power 48-dB/stage linear-in-dB variable gain amplifier for direct-conversion receivers. Microelectronics Journal, 2012, vol. 43, no. 4, p. 274-279. DOI: 10.1016/j.mejo.2012.01.005

[12] KALENTERIDIS, V., VLASSIS, S., SISKOS, S. 1.5-V CMOS exponential current generator. Analog Integrated Circuits and Signal Processing, 2012, vol. 72, no. 2, p. 333-341. DOI: 10.1007/s10470-012-9836-5

[13] KAO, C., TSENG, C., HSIEH, C. Low-voltage exponential function converter. Circuits, Devices and Systems, 2005, vol. 152, no. 5, p. 485-487. DOI: $10.1049 /$ ip-cds: 20045110

[14] DE LA CRUZ BLAS, C., LÓPEZ-MARTÍN, A. Novel low-power high-dB range CMOS pseudo-exponential cells. ETRI Journal, 2006, vol. 28, no. 6, p. 732-738. DOI: 10.4218/etrij.06.0106.0121

[15] KABOLI, M., GHANAVATI, B., AKHLAGHI, M. A new CMOS pseudo approximation exponential function generator by modified particle swarm optimization algorithm. Integration, the VLSI Journal, 2017, vol. 56, p. 70-76. DOI: 10.1016/j.vlsi.2016.10.003

[16] MORO-FRIAS, D., DE LA CRUZ BLAS, C., SANZ-PASCUAL, T. PWL current-mode CMOS exponential circuit based on maximum operator. IEEE Transactions on Circuits and Systems II: Express Brief, 2015, vol. 62, no. 12, p. 1169-1173. DOI: 10.1109/TCSII.2015.2468972

[17] MISENER, R. C., FLOUDAS, A. Piecewise-linear approxima- tions of multidimensional functions. Journal of Optimization Theory and Applications, 2010, vol. 145, no. 1, p. 120-147. DOI: $10.1007 / \mathrm{s} 10957-009-9626-0$

[18] ABUELMATTI, T., ABUELMATI, A. A new current mode CMOS analog programmable arbitrary nonlinear function synthesizer. Microelectronics Journal, 2012, vol. 43, no. 11, p. 802-8. DOI: 10.1016/j.mejo.2012.07.003

[19] SCHIFER, V., EVANS, W. A. Approximations in sinewave generation and synthesis. Radio and Electronic Engineer, 1978, vol. 48, no. 3, p. 113-121. DOI: 10.1049/ree.1978.0016

[20] POPA, C. Low-voltage CMOS current-mode exponential circuit with $70 \mathrm{~dB}$ output range. Microelectronics Journal, 2013, vol. 44, no. 12 , p. 1348-1357. DOI: 10.1016/j.mejo.2013.09.005

[21] NADERI SAATLO, A., OZOGUZ, S. A new CMOS exponential circuit with extended linear output range. In Proceedings of the 20th European Conference on Circuit Theory and Design (ECCTD)., Linkoping (Sweden), 2011, p. 893-896. DOI: 10.1109/ECCTD.2011.6043814

[22] NADERI, A., KHOEI, A., HADIDI, K. H., et al. A new high speed and low power four-quadrant CMOS analog multiplier in current-mode. AEU-International Journal of Electronics and Communications, 2009, vol. 63, no. 9, p. 769-775. DOI: 10.1016/j.aeue.2008.06.002

[23] FILANOVSKY, M., ALLAM, A. Mutual compensation of mobility and threshold voltage temperature effects with applications in CMOS circuits. IEEE Transactions on Circuits and Systems I: Fundamental Theory and Applications, 2001, vol. 48, no. 7,p. $876-884$. DOI: $10.1109 / 81.933328$

[24] BULT, K., WALlingA, H. A class of analog CMOS circuits based on the square-law characteristic of an MOS transistor in saturation. IEEE Journal of Solid-State Circuits, 1997, vol. 2, no. 3, p. 357-365. DOI: 10.1109/JSSC.1987.1052733

[25] LIU, H., BOON, C. C., HE, X. F., et al A wideband analogcontrolled variable-gain amplifier with dB-linear characteristic for high-frequency applications. IEEE Transactions on Microwave Theory and Techniques, 2016, vol. 64, no. 2, p. 533-540. DOI: 10.1109/TMTT.2015.2513403

[26] SÁNCHEZ-RODRIGUEZ, T., GALÁN, A., PEDRO, M., et al. Low-power CMOS variable gain amplifier based on a novel tunable transconductor. IET Circuits, Devices and Systems, 2015, vol. 9 , no. 2 , p. 105-110. DOI: 10.1049 /iet-cds.2014.0130

\section{About the Authors ...}

Ali NADERI SAATLO received his B.Sc. degree in Communication Engineering from Urmia Azad University, in 2005, the M.Sc. degree in Electrical Engineering from Urmia University, Urmia, Iran in 2008, and the Ph.D. in Electronics Engineering from Istanbul Technical University, Turkey in 2014. Since 2011, he has been an assistant professor in Electrical Engineering Dept. of Urmia Azad University. His research interests are analog integrated circuit design for fuzzy applications, high performance analog circuits, and digital signal processing.

Serdar OZOGUZ received his BSEE, MSEE and PhD degrees in Electronics Engineering from Istanbul Technical University in 1991, 1993 and 2000 respectively. Since 2009 , he is working as a full professor in Istanbul Technical University. His research interests include analog circuit design, chaotic circuits and chaos applications. Serdar Ozoguz has become a recipient of the Young Scientist Award of the Scientific and Technical Research Council of Turkey in 2004 and the Outstanding Young Scientist Award of the Turkish Academy of Sciences in 2002. 